\title{
High resolution mass spectrometry based profiling of diet-related deoxyribonucleic acid adducts
}

\author{
Lieselot Y. Hemeryck a , Anneleen I. Decloedt a, Julie Vanden Bussche a , Karen P. Geboes ${ }^{\text {b }}$, \\ Lynn Vanhaecke ${ }^{\text {a, * }}$ \\ a Laboratory of Chemical Analysis, Department of Veterinary Public Health and Food Safety, Faculty of Veterinary Medicine, Ghent University, \\ Salisburylaan 133, Merelbeke, 9820, Belgium \\ ${ }^{\mathrm{b}}$ Division of Digestive Oncology, Department of Gastroenterology, University Hospital Ghent, De Pintelaan 185, Ghent, 9000, Belgium
}

\section{H I G H L I G H T S}

- An UHPLC-HRMS/MS method using Quadrupole-Orbitrap technology was developed for the detection of dietrelated DNA adducts.

- A diet-related DNA adduct database was constructed.

- The method was validated and both the method and database were applied in vitro and in vivo.

- Targeted and untargeted diet-related DNA adducts were detected in in vitro and in vivo DNA samples.

- The method and database will help investigate the role of diet-related DNA adducts in diet-related chronic diseases.

\section{A R T I C L E I N F O}

Article history:

Received 1 June 2015

Accepted 7 August 2015

Available online 22 August 2015

\section{Keywords:}

Colorectal cancer

Dietary chemicals

Deoxyribonucleic acid adduct database

High resolution mass spectrometry

Quadrupole-Orbitrap
G R A P H I C A L A B S T R A C T

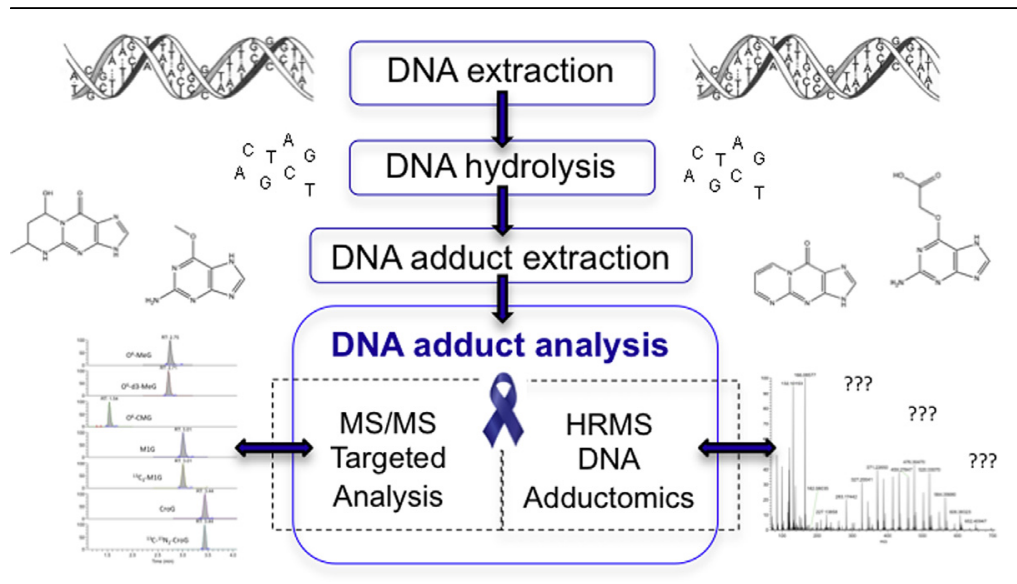

\begin{abstract}
A B S T R A C T
Exposure of DNA to endo- and exogenous DNA binding chemicals can result in the formation of DNA adducts and is believed to be the first step in chemically induced carcinogenesis. DNA adductomics is a relatively new field of research which studies the formation of known and unknown DNA adducts in DNA due to exposure to genotoxic chemicals. In this study, a new UHPLC-HRMS(/MS)-based DNA adduct detection method was developed and validated. Four targeted DNA adducts, which all have been linked to dietary genotoxicity, were included in the described method; $\mathrm{O}^{6}$-methylguanine $\left(\mathrm{O}^{6}\right.$-MeG), $\mathrm{O}^{6}$-carboxymethylguanine $\left(\mathrm{O}^{6}-\mathrm{CMG}\right)$, pyrimidopurinone (M1G) and methylhydroxypropanoguanine (CroG). As a supplementary tool for DNA adductomics, a DNA adduct database, which currently contains 123 different diet-related DNA adducts, was constructed. By means of the newly developed method and database, all 4 targeted DNA adducts and 32 untargeted DNA adducts could be detected in different DNA samples. The obtained results clearly demonstrate the merit of the described method for both targeted and untargeted DNA adduct detection in vitro and in vivo, whilst the diet-related DNA adduct database can distinctly facilitate data interpretation.
\end{abstract}

(C) 2015 Elsevier B.V. All rights reserved.

\footnotetext{
* Corresponding author.

E-mail address: Lynn.Vanhaecke@ugent.be (L. Vanhaecke).
} 


\section{Introduction}

When a genotoxic chemical binds to DNA, chemically stable DNA adducts can be introduced to the DNA sequence. Carcinogenesis is known to arise due to a combination of both genetic and environmental factors, and DNA adduct formation is considered to be the first step in chemically induced carcinogenesis [1,2]. Different 'endogenous' DNA adduct types are formed continuously due to normal physiological processes like oxidative stress and inflammation. The same and also other DNA adduct types can be formed due to exposure to environmental chemicals, which results in the occurrence of 'exogenous' DNA adduct levels [3].

It has been demonstrated that exogenous DNA adduct formation causally links the consumption of aflatoxin B1 contaminated food to hepatocellular carcinoma incidence [4] and more recently, researchers also established a causal link between the smoking of tobacco, the formation of PAH-DNA adducts ( $\mathrm{PAH}=$ polycyclic aromatic hydrocarbons) and cervical cancer [5]. In consequence, research on DNA adduct related carcinogenesis due to certain environmental factors has gained an intelligible interest during the past decade.

Colon cancer incidence is mainly influenced by the presence (or absence) of particular environmental factors, as literature states that up to $70 \%$ of colon cancer cases could be prevented by dietary changes [6,7]. For example, in developed ('Western') countries, high red and processed meat consumption has been linked to a significantly higher colon cancer incidence in both men and women. Different hypotheses have been put forward to substantiate this meat-cancer relationship. The two remaining hypotheses however, discuss the role of $N$-nitroso compound (NOC) and aldehyde formation in the human gut upon digestion of red and processed meat [8]. Both NOCs and aldehydes can bind to DNA nucleobases, resulting in the possible formation of a multitude of DNA adducts $[6,9]$.

To study the role of meat consumption in particular, and the human diet and exosphere in general, in the initiation and progression of cancer, the development of a highly specific and sensitive method for the detection of diet-related DNA adducts could prove very useful.

Different analytical methods such as immunoassays, immunohistochemistry, ${ }^{32}$ P-postlabeling, GC- or HPLC-ECD, HPLC-FD, GCor (HP)LC-MS, (LC-)NMR, LM-PCR and AMS [10,11] have been developed for the detection of specific or bulky DNA adducts. Although every analytical method has its advantages, MS may prove to be the most useful analytical method for the screening, detection and quantification of diet-related DNA adducts [11,12]. The most widely used DNA adduct detection technique is ${ }^{32} \mathrm{P}-$ postlabeling, which has excellent sensitivity, but lacks adequate specificity [13]. Ultra-High Performance Liquid Chromatography (UHPLC) combined with High Resolution MS (HRMS) detection techniques on the other hand, enable accurate identification of analytes based on chemical composition and exact compound mass. Furthermore, MS techniques can also provide structural information and due to ongoing technical improvements, MS currently offers an excellent qualitative and quantitative tool for DNA adduct research [10,14-16]. Although the advantages of HRMS are clear, it may lack sufficient sensitivity for quantification of low levels of DNA adducts. An alternative approach is the use of MS/MS, which most often brings about lower detection limits, enabling a more accurate quantitation. A recent advance in MS technology now combines the specificity of HRMS and the sensitivity of triple quadrupole MS/MS by combining both methodologies in 'hybrid quadrupole MS' systems [17,18]. In other words, MS can easily measure low DNA adduct levels with the highest specificity, enabling simultaneous identification and quantification of different compounds.

In light of these advances, the possibilities of a hybrid HRMS/MS approach was investigated through the development and validation of a method capable of quantifying 4 DNA adducts that may be related to the meat and colon cancer hypothesis: $0^{6}$-methylguanine $\left(\mathrm{O}^{6}-\mathrm{MeG}\right), \mathrm{O}^{6}$-carboxymethylguanine $\left(\mathrm{O}^{6}-\mathrm{CMG}\right)$, pyrimido [1,2-a]purin-10(1H)-one (M1G) and $\alpha$-methyl- $\gamma$-hydroxy-1, $\mathrm{N}_{2-}$ propanoguanine ( $\mathrm{CroG}$ ). The formation of $\mathrm{O}^{6}-\mathrm{MeG}$ and $\mathrm{O}^{6}-\mathrm{CMG}$ has been linked to NOC formation in the gut during meat digestion [6], while M1G and CroG can be produced when diet-related or endogenously formed malondialdehyde or crotonaldehyde (both lipid peroxidation products) bind to guanine ( $G$ ) in DNA [9]. Besides the described targeted approach (UHPLC-HRMS/MS), an untargeted full scan UHPLC-HRMS approach was explored to enable future DNA adductomics research. In addition, to facilitate DNA adduct research, known diet-related DNA adducts were defined and listed in an in-house database.

\section{Materials and methods}

\subsection{Reagents and chemicals}

Standards of M1G, CrodG ( $\alpha$-methyl- $\gamma$-hydroxy-1, $N_{2}$-propano- $2^{\prime}-$ deoxyguanosine) and their internal standards $\mathrm{M} 1 \mathrm{G}-{ }^{13} \mathrm{C}_{3}$ and CrodG ${ }^{13} \mathrm{C}^{15} \mathrm{~N}_{2}$ were obtained from Toronto Research Chemicals (Toronto, Canada). $\mathrm{O}^{6}$-CMdG $\left(\mathrm{O}^{6}\right.$-carboxymethyl-2'-deoxyguanosine) was kindly provided by Prof. S. Moore (Liverpool John Moores University, UK), whilst $\mathrm{O}^{6}$-MedG ( $\mathrm{O}^{6}$-methyl-2'-deoxyguanosine), $\mathrm{O}^{6}-\mathrm{d} 3-M e d G$ and a guanine $(\mathrm{G})$ standard were purchased at SigmaAldrich (St. Louis, USA).

Ethyldiazoacetate (EtDA) (the precursor of potassium diazoacetate (KDA)), crotonaldehyde (CRO) and 1,1,3,3-tetramethoxypropane (the precursor of malondialdehyde (MDA) were obtained from SigmaAldrich (St. Louis, USA) as well.

\subsection{Preparation of stock and working solutions}

Prior to preparation of stock and working solutions, $\mathrm{O}^{6}-\mathrm{CMdG}$, $\mathrm{O}^{6}$-MedG, $\mathrm{O}^{6}$-d3-MedG, CrodG and CrodG $-{ }^{13} \mathrm{C}^{15} \mathrm{~N}_{2}$ were hydrolyzed from guanosines (nucleotides) to guanines (nucleobases) in $0.1 \mathrm{M}$ formic acid at $80{ }^{\circ} \mathrm{C}$ during $30 \mathrm{~min}$ [19]. All standards were diluted in $\mathrm{MeOH}$ and stored $\left(-20^{\circ} \mathrm{C}\right)$ in stock and working solutions of respectively $500 \mathrm{ng} \mu \mathrm{L}^{-1}$ and $5 \mathrm{ng} \mu \mathrm{L}^{-1}$.

An $800 \mathrm{mM}$ stock solution of KDA was synthesized through alkaline hydrolysis of EtDA [20]. After further dilution with phosphate buffered saline (PBS), a working solution of $20 \mathrm{mM}$ KDA was obtained (Caution!: KDA is highly toxic and carcinogenic).

CRO was diluted to a stock and working solution of 800 and $20 \mathrm{mM}$ in PBS (Caution! CRO is highly toxic and carcinogenic). Stock and working solutions of both KDA and CRO were stored in dark glass bottles at $-80^{\circ} \mathrm{C}$.

The precursor of MDA; 1,1,3,3-tetramethoxypropane was diluted in acidified $(\mathrm{HCl}, \mathrm{pH} 2)$, deionized water and kept at $45{ }^{\circ} \mathrm{C}$ during $1 \mathrm{~h}$ to ensure full release of MDA. Working solutions of MDA (20 $\mathrm{mM}$ ) were freshly prepared prior to each experiment.

\subsection{DNA hydrolysis and DNA adduct purification}

DNA adducts were extracted and purified according to the protocol of Vanden Bussche et al. [19], during which DNA samples are initially subjected to DNA hydrolysis $\left(30 \mathrm{~min}, 80^{\circ} \mathrm{C}\right)$ in $0.1 \mathrm{M}$ formic acid to cleave both adducted and non-adducted DNA nucleobases from the DNA sequence. This is then followed by purification and sample cleanup by means of solid-phase extraction (SPE) (Oasis ${ }^{\circledR}$ HLB cartridges (1 cc, $30 \mathrm{mg}$ ) Waters (Milford, USA)). 
After SPE, the collected samples were evaporated to dryness under vacuum $\left(90 \mathrm{~min}, 20^{\circ} \mathrm{C}\right.$ ) and resuspended in $100 \mu \mathrm{L}$ of mobile phase. The obtained DNA adduct samples were stored at $-20^{\circ} \mathrm{C}$ until LCMS analysis.

\subsection{UHPLC-HRMS(/MS) analysis}

Chromatographic separation of DNA adducts was carried out by reversed phase chromatography. To ensure an optimal chromatographic separation and resolution, several column types were tested, including Nucleodur ISIS $(5 \mu \mathrm{m} 3 \times 150 \mathrm{~mm}$ and $4 \times 250 \mathrm{~mm})$ and Pyramid $(1.8 \mu \mathrm{m} 2 \times 100 \mathrm{~mm})$ (Machery-Nagel, Düren, Germany), Hypersil Gold $(1.9 \mu \mathrm{m} 2.1 \times 100 \mathrm{~mm}$, Thermo Fisher Scientific, San José, USA), Luna $\mathrm{NH}_{2}(5 \mu \mathrm{m} 4.60 \times 150 \mathrm{~mm}$, Phenomenex, Torrance, USA), Acquity BEH C18 (1.7 $\mu \mathrm{m}$ $2.1 \times 100 \mathrm{~mm})$, HSS C18 $(1.8 \mu \mathrm{m} 2.1 \times 100 \mathrm{~mm})$ and HSS T3 $(1.8 \mu \mathrm{m}$ $2.1 \times 100 \mathrm{~mm}$, Waters, Milford, MA, USA). Different mobile phases containing different percentages of solvent modifiers including acetic or formic acid and ammonium bicarbonate, and solvents i.e. water, methanol and acetonitrile were tested in parallel, and were pumped at $300 \mu \mathrm{L}$ per min by an Accela 1250 pump coupled to an Accela Autosampler (Thermo Scientific, San José, USA).

MS analysis was performed on a hybrid Quadrupole-Orbitrap High Resolution Accurate Mass Spectrometer (HRAM, Q-Exactive ${ }^{\mathrm{TM}}$, Thermo Fisher Scientific, San José, USA) coupled to a heated elektrospray ionization (HESI-II) source. All HESI and HRMS(/MS) parameters were optimized for the 4 targeted compounds and their internal standards. Chemical composition of the studied DNA adducts, theoretical and measured masses, observed mass deviation, monitored MS/MS ions and expected retention time (RT) are shown in Table 1.

General instrument control and data processing were performed with Xcalibur ${ }^{\mathrm{TM}} 3.0$ and ToxID ${ }^{\mathrm{TM}}$ software (Thermo Fisher Scientific, San José, USA).

\subsection{Validation in Calf Thymus DNA}

Currently, guidelines or regulations for the validation of analytical methods for the detection of DNA adducts in biological matrices are not available. The US Food and Drug Administration (FDA) did announce its intent to publish guidelines for biomarker detection methods, but a final draft has not yet been published [21]. Therefore, the guidelines for chemical methods described by the US FDA [22] and the EU Commission Directive 2002/657/EC [23] on the performance of analytical methods were taken into account. The described analytical method was validated by assessment of specificity, selectivity, linearity, precision and trueness with full scan HRMS. Quantification and detection limits were assessed with and for the full scan HRMS, SIM-HRMS and SIM-HRMS/MS method. The matrix in which all validation parameters were tested, consisted of the commercially available Calf Thymus DNA (CT-DNA). Lyophilized
CT-DNA was purchased from Rockland (Gilbertsville, Pennsylvania, USA) and stored at $4{ }^{\circ} \mathrm{C}$ in Tris-EDTA buffer $\left(1 \mathrm{mg} \mathrm{mL}^{-1}\right)$.

\subsection{DNA adducts in CT-DNA treated with genotoxic compounds}

The genotoxic compounds KDA, MDA and CRO were supplemented to CT-DNA in triplicate and incubated overnight $\left(37^{\circ} \mathrm{C}\right)$ to allow interaction and subsequent DNA adduct formation. KDA was added to $100 \mu \mathrm{g}$ of DNA in 3 different concentrations $(1 \mathrm{mM}$, $2.5 \mathrm{mM}$ and $5 \mathrm{mM}$ ). Separately, both MDA and CRO were added to $100 \mu \mathrm{g}$ of DNA as well, but in concentrations of $0.1 \mathrm{mM}, 0.25 \mathrm{mM}$ and $0.5 \mathrm{mM}$. After overnight incubation, DNA adducts were extracted and analyzed. An equal amount of CT-DNA $(100 \mu \mathrm{g})$, not treated with genotoxic compounds, was used as a negative control and to correct for intrinsically present DNA adduct levels.

\subsection{DNA adduct profiling in colon biopsies}

Left colon tumors were obtained during colonoscopy of 10 individuals diagnosed with colon cancer at the Ghent University Hospital. The average age of the patients was 74 , the youngest being 58 and the eldest 83. The group consisted of 7 men and 3 women. All resected samples were poorly to moderately differentiated primary adenocarcinomas which were submerged and stored in $95 \%$ of ethanol at $-80^{\circ} \mathrm{C}$. Two samples were retrieved in 2006 and 2007, but most were gathered in 2012 and 2013.

A Qiagen blood \& tissue kit (Qiagen, Hilden, Germany) was used for the extraction of DNA from the tissue samples according to the protocol provided by the manufacturer. The concentration and purity of the extracted DNA was determined with a Nanodrop ND1000 spectrophotometer (Isogen Lifescience, Ijsselstein, The Netherlands). Following this, the obtained DNA in each sample was hydrolyzed and DNA adducts were purified according to the protocol described under '2.3 DNA hydrolysis and DNA adduct purification'.

\section{Results}

\subsection{UHPLC-HRMS(/MS) settings optimization}

The Acquity BEH C18 Waters column and the use of $0.05 \%$ of acetic acid in water and $100 \% \mathrm{MeOH}$ as mobile phases provided optimal results for baseline peak separation, signal to noise ratio, peak area, peak shape and RT of all eluting targeted DNA adducts. The amount of $\mathrm{MeOH}$ was increased linearly from $0.85 \mathrm{~min}$, reaching $50 \%$ after $4 \mathrm{~min}$, which was then immediately followed by $1 \mathrm{~min}$ of $100 \% \mathrm{MeOH}$. For the remaining $2 \mathrm{~min}$, the column was reequilibrated at 95:5 0.05\% acetic acid in water:MeOH.

Three different MS acquisition modes were assessed: full scan MS, SIM-MS (selected ion monitoring) and SIM-MS/MS. Both negative and positive ionization mode (polarity switching) were

Table 1

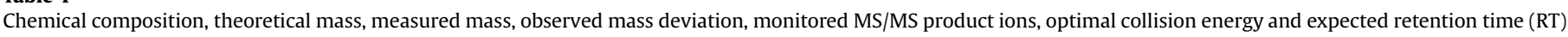
of the studied DNA adducts.

\begin{tabular}{|c|c|c|c|c|c|c|c|}
\hline Name & Chemical formula & $\begin{array}{l}\text { Theoretical mass } \\
\left(\mathrm{H}^{+}\right)\end{array}$ & $\begin{array}{l}\text { Measured mass } \\
\left(\mathrm{H}^{+}\right)\end{array}$ & $\begin{array}{l}\text { Mass deviation } \\
(\mathrm{ppm})\end{array}$ & $\begin{array}{l}\text { Monitored MS/MS } \\
\text { product ion }\left(\mathrm{H}^{+}\right)\end{array}$ & $\begin{array}{l}\text { MS/MS collision } \\
\text { energy (NCE) }\end{array}$ & $\begin{array}{l}\text { RT } \\
(\min )\end{array}$ \\
\hline $\mathrm{O}^{6}-\mathrm{CMG}$ & $\mathrm{C}_{7} \mathrm{H}_{7} \mathrm{~N}_{5} \mathrm{O}_{3}$ & 210.06217 & 210.06194 & 1.09 & 152.05663 & 40 & 1.5 \\
\hline $\mathrm{O}^{6}-\mathrm{MeG}$ & $\mathrm{C}_{6} \mathrm{H}_{7} \mathrm{~N}_{5} \mathrm{O}$ & 166.07234 & 166.07225 & 0.54 & 134.04613 & 60 & 2.8 \\
\hline d3-MeG & $\mathrm{C}_{6} \mathrm{H}_{4}\left[{ }^{2} \mathrm{H}\right]_{3} \mathrm{~N}_{5} \mathrm{O}$ & 169.09117 & 169.09100 & 1.01 & 134.04617 & 60 & 2.7 \\
\hline M1G & $\mathrm{C}_{8} \mathrm{H}_{5} \mathrm{~N}_{5} \mathrm{O}$ & 188.05669 & 188.05649 & 1.06 & 97.04008 & 60 & 3.0 \\
\hline${ }^{13} \mathrm{C}_{3}-\mathrm{M} 1 \mathrm{G}$ & $\mathrm{C}_{5}\left[{ }^{13} \mathrm{C}_{3} \mathrm{H}_{5} \mathrm{~N}_{5} \mathrm{O}\right.$ & 191.06675 & 191.06655 & 1.05 & 100.05006 & 60 & 3.0 \\
\hline CroG & $\mathrm{C}_{9} \mathrm{H}_{11} \mathrm{~N}_{5} \mathrm{O}_{2}$ & 222.09855 & 222.09843 & 0.54 & 152.05670 & 40 & 3.5 \\
\hline${ }^{13} \mathrm{C}^{15} \mathrm{~N}_{2}-\mathrm{CroG}$ & $\mathrm{C}_{8}\left[{ }^{13} \mathrm{C}\right]\left[{ }^{15} \mathrm{~N}\right]_{2} \mathrm{H}_{11} \mathrm{~N}_{3} \mathrm{O}_{2}$ & 225.09598 & 225.09560 & 1.69 & 155.05392 & 40 & 3.5 \\
\hline
\end{tabular}


included to not only enable the targeted analysis of the 4 targeted DNA adducts, but also the future analysis of all untargeted DNA adducts of interest, thus facilitating the use of this method as an "omics" tool. Likewise, the full MS scan range was held at $70-700 \mathrm{~m} / \mathrm{z}$ to enable an untargeted analysis next to the targeted analysis. A scan range of $100-250 \mathrm{~m} / \mathrm{z}$ was chosen for the targeted analysis in SIM-MS and SIM-MS/MS. Ultimately, the chosen scan resolution for full MS was 'ultra high' (100,000 FWHM (Full Width Half Maximum)), using 3 microscans. Optimal maximum inject time was $500 \mathrm{~ms}$ and the automatic gain control target was best operated in 'high dynamic range' $\left(3 \times \mathrm{e}^{6}\right)$. Sheath gas, auxiliary gas and sweep gas flow rate were set at 35,5 and 2 arbitrary units. Optimal spray voltage, capillary temperature, capillary voltage and heater temperature were kept at $4 \mathrm{kV}, 280^{\circ} \mathrm{C}, 40 \mathrm{~V}$ (positive or negative, dependent on polarity switch) and $330^{\circ} \mathrm{C}$ respectively. Slens RF-level was set at 90 and the same settings were applied for all acquisition types. However, a resolution of 70,000 and 17,500 FWHM appeared sufficient for respectively SIM-MS and SIM-MS/ MS, whilst a maximum inject time of $250 \mathrm{~ms}$ was applied. Normalized collision energy (NCE) and monitored product ions in MS/MS for each targeted DNA adduct and their internal standards are shown in Table 1. A chromatogram and full scan mass spectrum of all targeted compounds and their internal standards are presented in Figs. 1 and 2.

\subsection{Validation of the full HRMS method for the targeted detection of DNA adducts}

\subsubsection{Specificity}

The specificity of the HRMS method for the detection of $\mathrm{O}^{6}$ CMG, $\mathrm{O}^{6}-\mathrm{MeG}$ and $\mathrm{CroG}$ was assessed by analyzing over 21 blank CT-DNA samples and over 108 spiked CT-DNA samples. Spiked samples were fortified with a mixture of all DNA adducts, or each DNA adduct separately at three different concentrations levels $\left(2.50,5.00\right.$ and $7.50 \mathrm{ng} \mathrm{mL}^{-1}$ for $\mathrm{O}^{6}-\mathrm{CMG}$ and $\mathrm{O}^{6}-\mathrm{MeG} ; 0.25,0.50$ and $0.75 \mathrm{ng} \mathrm{mL}^{-1}$ for CroG). Comparison of the obtained chromatograms of blank and spiked DNA samples demonstrated that the DNA matrix did not cause interference for $\mathrm{O}^{6}-\mathrm{CMG}, \mathrm{O}^{6}-\mathrm{MeG}$ and $\mathrm{CroG}$ detection. In addition, spiking of $\mathrm{O}^{6}-\mathrm{CMG}, \mathrm{O}^{6}-\mathrm{MeG}$ and CroG lead to a significant increase in peak intensity with a signalto-noise ratio $>3$. For the M1G DNA adduct, specificity could not be thoroughly assessed by comparison of blank and fortified samples, since the matrix DNA contained endogenous levels of M1G. However, no other matrix substances significantly interfered with the analysis of M1G (signal-to-noise ratio >3) and spiking of three different levels of M1G $(0.25,0.50$ and $0.75 \mathrm{ng} \mathrm{mL}^{-1}$ ) to CT-DNA did significantly increase the M1G peak area at the expected RT.

\subsubsection{Selectivity}

The methods' selectivity could be demonstrated by analyte identification based on relative RT (ratio of analyte RT and corresponding internal standard RT) and the accurate mass of their positive ions $\left([\mathrm{M}+\mathrm{H}]^{+}\right)$. Only chromatographic peaks of interest with signal-to-noise ratios that exceeded 3 were taken into account and tolerance levels for relative RT and maximum mass deviation were established at $2.5 \%$ and $5 \mathrm{ppm}$ respectively.

\subsubsection{Linearity}

Linearity was assessed by deploying 2 separate calibration curves in triplicate. A first calibration curve was established in a low range that would enable quantification of endogenous and exogenous levels of DNA adducts $[3,24]$, whereas the second calibration curve was used to enable the in vitro application of this method (see "DNA adducts in CT-DNA treated with genotoxic compounds"). For

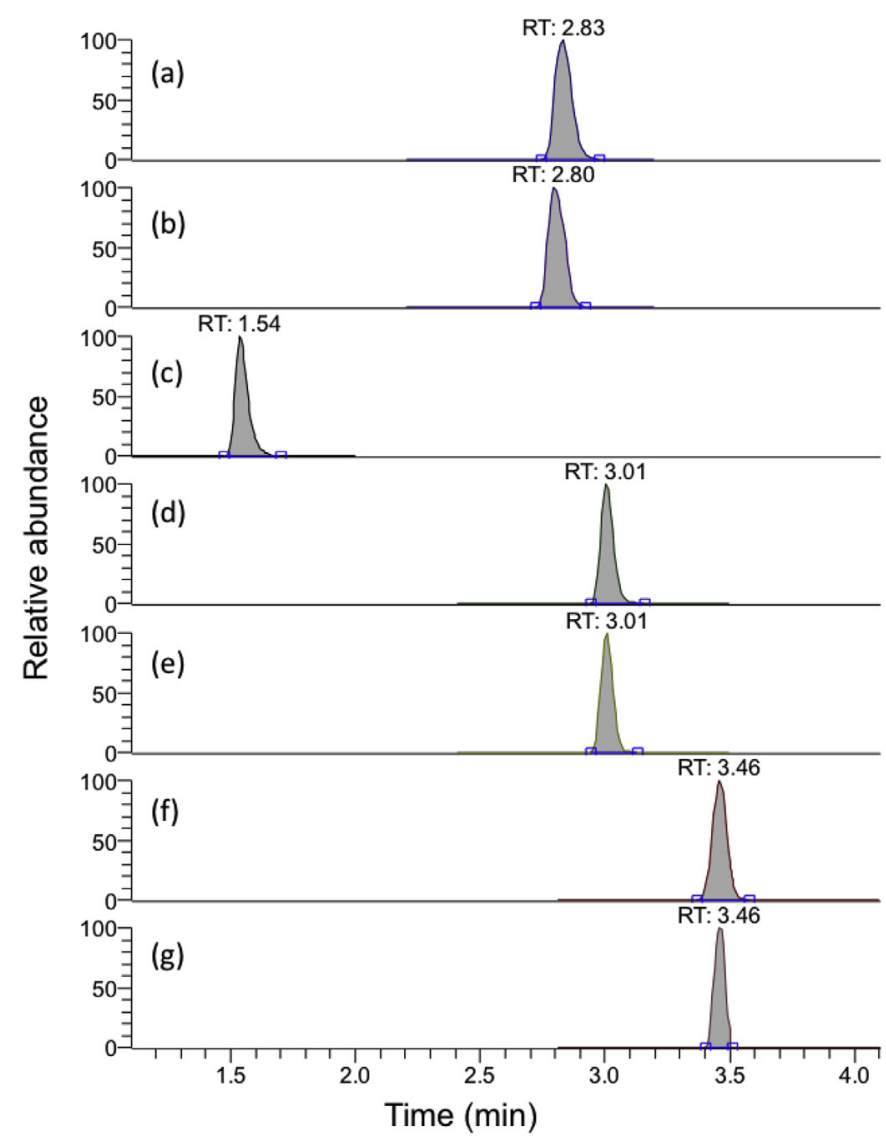

Fig. 1. Chromatogram of $\mathrm{O}^{6}-\mathrm{MeG}$ (a), d3-MeG (b), $\mathrm{O}^{6}-\mathrm{CMG}$ (c), $\mathrm{M} 1 \mathrm{G}$ (d), ${ }^{13} \mathrm{C}_{3}-\mathrm{M} 1 \mathrm{G}$ (e), CroG (f) and ${ }^{13} \mathrm{C}^{15} \mathrm{~N}_{2}-\mathrm{CroG}(\mathrm{g})$ in CT-DNA with SIM-MS/MS acquisition.

the calibration curves, the matrix DNA was fortified with 0.025 ; $0.05 ; 0.075 ; 0.1 ; 0.125 ; 0.25 ; 0.50 ; 0.75 ; 1 ; 1.25 ; 2.5 ; 5 ; 7.5 ; 10$ and $12.5 \mathrm{ng} \mathrm{mL}^{-1} \mathrm{O}^{6}-\mathrm{CMG}, \mathrm{O}^{6}-\mathrm{MeG}, \mathrm{M} 1 \mathrm{G}$ and CroG. The obtained coefficients of determination $\left(R^{2}\right)$ proved to be over 0.99 , suggesting a good to excellent linearity, which could be confirmed by means of a one-way ANOVA (F-test) linearity test $(\mathrm{P}>0.05)$. The ANOVA model was constructed with the detected area ratio of each component (DNA adduct peak area/area of the internal standard) as the dependent variable and the calibration curve concentration levels as the independent variable (SPSS Statistics 21).

\subsubsection{Mean recovery}

To assess mean recovery of the targeted compounds, 3 series of six replicates of the earlier described CT-DNA samples fortified with three different spike levels were utilized. As M1G appeared to be endogenously present in CT-DNA, total concentrations were adjusted to the measured fortified concentrations prior to calculation of the corrected mean recovery. Mean recovery of M1G, CroG and $\mathrm{O}^{6}-\mathrm{MeG}$ were within the narrow range of 97 and $104 \%$ and thus proved to be excellent. $\mathrm{O}^{6}-\mathrm{CMG}$ mean recoveries ranged between 89 and $108 \%$, which is still well within the acceptable range of $80-120 \%$.

\subsubsection{Precision}

To evaluate precision, repeatability and intra-lab reproducibility were determined by calculation of the relative standard deviations (RSD\%). Three sets of six fortified samples (equals the three fortification levels with six replicates each) were analyzed to test repeatability. One additional set of six samples fortified, 


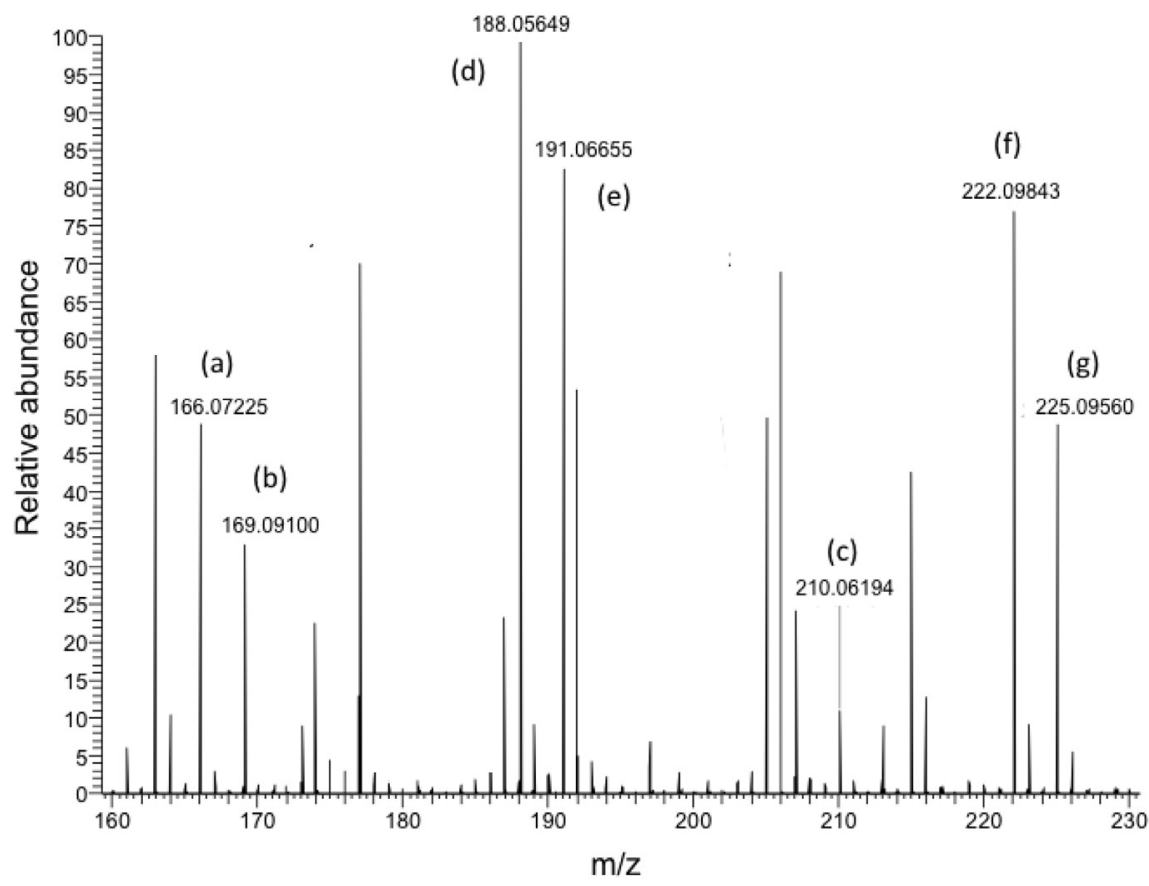

Fig. 2. Mass spectrum of $\mathrm{O}^{6}-\mathrm{MeG}(\mathrm{a}), \mathrm{d} 3-\mathrm{MeG}(\mathrm{b}), \mathrm{O}^{6}-\mathrm{CMG}$ (c), $\mathrm{M} 1 \mathrm{G}(\mathrm{d}),{ }^{13} \mathrm{C}_{3}-\mathrm{M} 1 \mathrm{G}$ (e), CroG (f) and ${ }^{13} \mathrm{C}^{15} \mathrm{~N}_{2}-\mathrm{CroG}$ (g) in CT-DNA with HRMS acquisition.

extracted and analyzed by a different person at a different time enabled assessment of intra-lab reproducibility. As no detectable $\mathrm{O}^{6}$-CMG, $\mathrm{O}^{6}-\mathrm{MeG}$ and $\mathrm{CroG}$ levels appeared to be present in the matrix, and since endogenous levels in different tissues demonstrate a certain variation, fortification levels for $\mathrm{O}^{6}$-CMG were selected according to the estimated limit of detection and quantification with the full HRMS method. To establish M1G levels, the endogenous levels of M1G in the matrix DNA were taken into account. For CroG and $\mathrm{O}^{6}-\mathrm{MeG}$, spike levels were adjusted to those of $\mathrm{M} 1 \mathrm{G}$ and $\mathrm{O}^{6}-\mathrm{CMG}$ respectively, due to their analogue nature and origin. Also, for M1G, the endogenously present M1G DNA adduct levels in the CT-DNA were subtracted from the total measured M1G concentrations in spiked CT-DNA prior to calculation of the final/fortified concentration and RSD\%. For M1G, CroG and $\mathrm{O}^{6}$ MeG, repeatability appeared to be excellent since RSD\% consistently proved below $4 \%$ for each analyte and fortification level. In comparison, repeatability for $\mathrm{O}^{6}$-CMG was less good, but still below the acceptable RSD\% limit of 15 with a RSD\% of 9. Intra-lab reproducibility for all targeted compounds is good as well, with all RSD\% below 9 .

\subsubsection{Limits of detection and quantification}

LOQs were determined at a minimal signal-to-noise ratio of 10 , whereas limits of detection (LODs) required a signal-to-noise ratio of at least 3 (Table 2). LODs and LOQs with SIM-MS and SIM-MS/MS clearly overthrew the full HRMS LODs and LOQs for $0^{6}-\mathrm{CMG}$ and $\mathrm{O}^{6}$-MeG. For CroG and M1G, differences in LODs and LOQs between full HRMS, SIM-MS and SIM-MS/MS were less pronounced. Practical assessment of LOD and LOQs for M1G is limited by the presence of M1G levels inherent to the CT-DNA matrix. Therefore, average LOD and LOQs levels for M1G were assessed based on cochromatography of added M1G with endogenously present M1G in the CT-DNA matrix.

For the combined detection and quantification of all 4 targeted DNA adducts, SIM-MS/MS would be preferred over SIM-MS and full MS since it combines the lowest LOQs for all 4 targeted compounds.

\subsection{Development of a diet-related DNA adduct database to} facilitate interpretation of untargeted DNA adductomics studies

To construct an in-house database, literature was searched for DNA adducts of which harmful exogenous levels may be linked to the human diet. The main focus of the search were DNA adduct types originating from DNA alkylation or oxidation since these reaction types are of particular interest regarding the mechanisms behind colon cancer initiation and promotion by red and processed meat consumption $[8,25]$. The retrieved DNA adducts were assembled in a database which currently contains 123 different diet-related DNA adducts (Supporting information Table 1).

By means of ToxID ${ }^{\text {TM }}$ software (Thermo Fisher Scientific, San José, USA) and the self-constructed database, the full scan HRMS spectra of different DNA samples could be screened for the presence of non-targeted diet-related DNA adducts in retrospect. The considered inclusion criteria consisted of a minimal signal intensity of 10,000 , a maximum mass deviation of $10 \mathrm{ppm}$, recurrence and stable RT of the DNA adduct of interest in replicate and repeat samples and a $C^{12} / C^{13}$ ratio approaching the natural 99:1 ratio. Detection of a certain diet-related DNA adduct with full MS and Tox-ID ${ }^{\mathrm{TM}}$ profiling renders chromatograms and data on measured peak area of the masses of interest and can thus suggest the presence of a putative DNA adduct in a sample. If believed relevant, exact confirmation of DNA adduct identity can be made by use of commercially available analyte standards.

In this study, normalization of the obtained untargeted DNA adduct Tox-ID ${ }^{\mathrm{TM}}$ data was based on the measured signal intensity (area) of the guanine nucleobase in each sample by expressing DNA adduct area relative to guanine area. The identity of guanine was confirmed with an analytical standard.

To demonstrate the possible merit and application of the described approach, full HRMS spectra obtained from blank CTDNA, CT-DNA treated with KDA, MDA and CRO, and also tumor biopsies were interpreted by means of ToxID ${ }^{\mathrm{TM}}$ profiling and the self-constructed database. The results are discussed in the related sections below and documented in Fig. 3. 
Table 2

Obtained validation parameters for each targeted DNA adduct including mean recovery, repeatability, intra-lab reproducibility, LODs and LOQs.

\begin{tabular}{|c|c|c|c|c|c|c|c|}
\hline Analyte & $\begin{array}{l}\text { Spike levels } \\
\text { (ng per } 100 \\
\mu \mathrm{g} \text { DNA) }\end{array}$ & $\begin{array}{l}\text { Mean } \\
\text { recovery } \\
\pm \mathrm{SD}(\%)\end{array}$ & $\begin{array}{l}\text { Repeatability } \\
\mathrm{RSD} \pm \mathrm{SD}(\%)\end{array}$ & $\begin{array}{l}\text { Intra-lab } \\
\text { reproducibility } \\
\text { RSD (\%) }\end{array}$ & $\begin{array}{l}\text { LOD full MS } \\
\text { (adducts per } 10^{8} \\
\text { nucleotides) }\end{array}$ & $\begin{array}{l}\text { LOQ SIM } \\
\text { (adducts per } 10^{8} \\
\text { nucleotides) }\end{array}$ & $\begin{array}{l}\text { LOQ MS/MS } \\
\text { (adducts per } 10^{8} \\
\text { nucleotides) }\end{array}$ \\
\hline \multirow[t]{3}{*}{$\mathrm{O}^{6}-\mathrm{CMG}$} & 1.25 & $89.5 \pm 9.0$ & $8.4 \pm 4.8$ & 5.6 & 888 & 22.2 & 22.2 \\
\hline & 2.50 & $107.9 \pm 4.4$ & $7.0 \pm 2.9$ & 5.8 & & & \\
\hline & 3.75 & $106.6 \pm 3.1$ & $7.1 \pm 2.9$ & 8.3 & & & \\
\hline \multirow[t]{3}{*}{$\mathrm{O}^{6}-\mathrm{MeG}$} & 1.25 & $103.8 \pm 0.8$ & $1.7 \pm 0.2$ & 3.6 & 28.1 & 28.1 & 2.82 \\
\hline & 2.50 & $100.2 \pm 1.8$ & $2.5 \pm 0.4$ & 1.8 & & & \\
\hline & 3.75 & $101.6 \pm 1.5$ & $1.5 \pm 0.6$ & 2.1 & & & \\
\hline \multirow[t]{3}{*}{ M1G } & 0.125 & $103.3 \pm 0.3$ & $3.7 \pm 1.5$ & 4.4 & 2.48 & 2.48 & 4.96 \\
\hline & 0.250 & $97.3 \pm 1.9$ & $2.5 \pm 0.5$ & 6.9 & & & \\
\hline & 0.375 & $100.2 \pm 0.5$ & $2.1 \pm 0.7$ & 3.8 & & & \\
\hline \multirow[t]{3}{*}{ CroG } & 0.125 & $97.4 \pm 1.4$ & $2.2 \pm 0.8$ & 5.8 & 0.52 & 1.05 & 4.20 \\
\hline & 0.250 & $97.0 \pm 1.3$ & $2.8 \pm 0.9$ & 3.9 & & & \\
\hline & 0.375 & $98.0 \pm 2.0$ & $1.8 \pm 1.0$ & 3.6 & & & \\
\hline
\end{tabular}

\subsection{CT-DNA adducts}

The commercially available CT-DNA contained endogenous levels of M1G, whereas no detectable endogenous levels of $\mathrm{O}^{6}$ $\mathrm{MeG}, \mathrm{O}^{6}-\mathrm{CMG}$ and $\mathrm{CroG}$ could be detected.

Next to the targeted cyclic M1G DNA adduct, Tox-ID ${ }^{\text {TM }}$ profiling indicated the possible presence of 20 additional DNA adducts (Fig. 3). This includes M1A (oxopropenylA), M1C (oxopropenylC), $1, \mathrm{~N}_{2}$-ethenoG, non- $\mathrm{O}^{6}$-carboxymethylG, two non- $\mathrm{O}^{6}$-methylGs, carboxyethylG, FapyG (formamidopyrimidineG), FapyA (formamidopyrimidineA), methylT, dimethylT or ethylT, carboxymethylT, carboxyethylT, methoxymethylC, methoxymethylT, butylG, hydroxyethylG, propylG, trimethylG and hydroxyG DNA adducts (Fig. 3).

\subsection{DNA adducts in CT-DNA treated with genotoxic compounds}

Upon addition of different concentrations of KDA, MDA and CRO to CT-DNA, $\mathrm{O}^{6}-\mathrm{MeG}, \mathrm{O}^{6}-\mathrm{CMG}, \mathrm{M} 1 \mathrm{G}$ and CroG DNA adducts were formed during overnight incubation. $\mathrm{O}^{6}-\mathrm{CMG}$ and $\mathrm{O}^{6}-\mathrm{MeG}$ were formed upon addition of KDA, CroG was formed due to addition of CRO and M1G was produced after the addition of MDA; a clear dose-response effect was observed (Fig. 4). Based on the fact that lipid peroxidation products are highly reactive chemicals, and also based on practical assessment of the experimental setup, MDA and CRO concentration levels were adjusted to avoid overload of the LC and MS system. Therefore, the added MDA and CRO concentrations are lower than the added KDA concentrations.

Addition of KDA to DNA also resulted in the formation of untargeted DNA adducts (Fig. 3). A first observation was the possible presence of 2 additional methylG compounds other than $\mathrm{O}^{6}$-MeG at RTs of 1.7 and $2.3 \mathrm{~min}$. Both additional MeG DNA adducts were more prevalent than $\mathrm{O}^{6}$-MeG. In addition to $\mathrm{MeG}$, a slight increase in butylG, methoxymethylC and a significant increase in methylT and carboxymethylT could be noted after KDA treatment.

During screening of CT-DNA treated with MDA by means of the DNA adduct database, it could be observed that not only M1G, but also M1A and M1C levels appeared to increase in a dose-response manner when the MDA concentration was raised (Fig. 3). In addition, just like KDA, MDA conceivably promoted the formation of methoxymethylC.

Untargeted analysis of CT-DNA samples that had been treated with CRO revealed the probable presence of methoxymethylC,

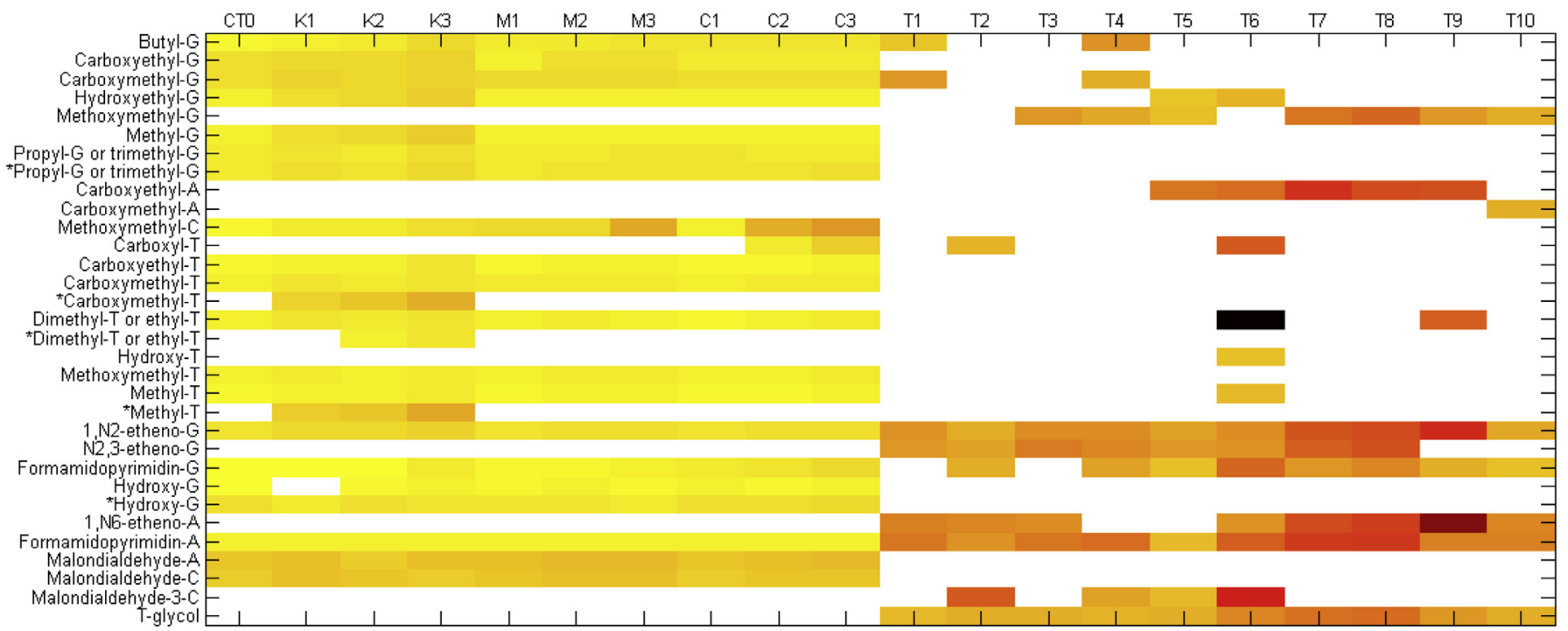

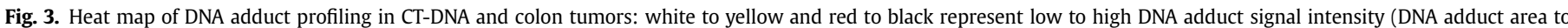

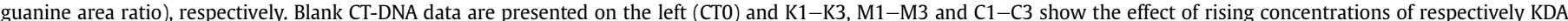

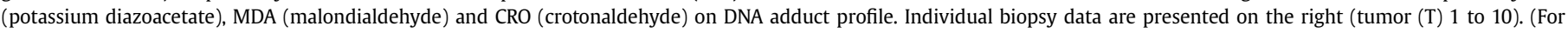
interpretation of the references to colour in this figure legend, the reader is referred to the web version of this article.) 


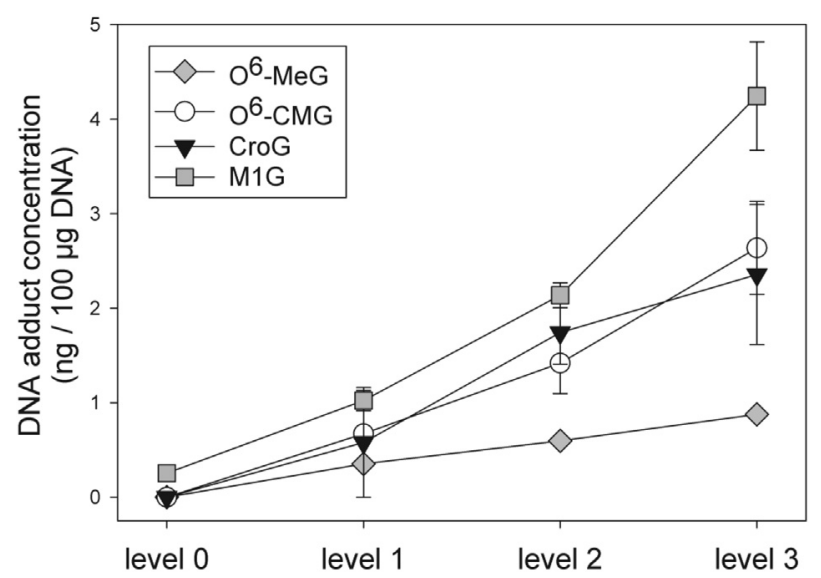

Fig. 4. Mean ( \pm sd) $\mathrm{O}^{6}-\mathrm{MeG}, \mathrm{O}^{6}-\mathrm{CMG}$, CroG and M1G DNA adduct formation in CT-DNA after exposure to genotoxic chemicals. Level 0 corresponds to the negative control samples. Level 1 represents addition of $1 \mathrm{mM}$ of KDA or $0.1 \mathrm{mM}$ of CRO or MDA. Level 2 equals addition of $2.5 \mathrm{mM}$ of KDA or $0.25 \mathrm{mM}$ of CRO or MDA. Level 3 demonstrates addition of $5 \mathrm{mM}$ KDA or $0.5 \mathrm{mM}$ of CRO or MDA.

FapyG, hydroxyG and carboxyT.

\subsection{DNA adducts in colon biopsies}

$\mathrm{O}^{6}$-CMG could be identified and quantified in 8 out of 10 tumor samples. For quantification of $\mathrm{O}^{6}-\mathrm{CMG}$ in each sample, the DNA adduct concentrations were adjusted according to the measured DNA concentration in each sample. The mean amount of $\mathrm{O}^{6}-\mathrm{CMG}$ equaled 815 adducts per $10^{8}$ nucleotides, with a relatively high interindividual variability (range from < LOD to 1630 adducts per $10^{8}$ nucleotides). M1G, CroG and $0^{6}-\mathrm{MeG}$ could not be detected.

Untargeted analysis of all tumor samples (T1-T10) was carried out in parallel. The list of observed DNA adducts includes both alkylation and oxidation DNA adducts, i.e. non- ${ }^{6}-\mathrm{CMG}$ (same mass as $\mathrm{O}^{6}$-CMG, but a different RT), butylG, hydroxyethylG, carboxyethylA, carboxymethylA, methylT, dimethylT or ethylT, carboxyT, FapyG, FapyA, Tglycol, hydroxyT, $1, \mathrm{~N}_{2}$-ethenoG, $\mathrm{N}_{2}, 3$-ethenoG, $1, \mathrm{~N}_{6^{-}}$ ethenoA, M3C and methoxymethylG (Fig. 3).

\section{Discussion}

A new UHPLC-HRMS/MS method was developed to enable targeted and untargeted detection of both known and unknown DNA adducts. A first focus was the separation and targeted detection of $\mathrm{O}^{6}-\mathrm{CMG}, \mathrm{O}^{6}-\mathrm{MeG}, \mathrm{M} 1 \mathrm{G}$ and CroG. These DNA adducts are of particular interest because DNA-damaging NOCs and lipid peroxidation products appear to be the two main culprits in the complex underlying mechanism that links red and processed meat consumption to an elevated colon cancer risk $[8,25]$. The combined targeted detection of $\mathrm{O}^{6}-\mathrm{CMG}, \mathrm{O}^{6}-\mathrm{MeG}, \mathrm{M} 1 \mathrm{G}$ and $\mathrm{CroG}$ is unique as it permits simultaneous investigation of 2 important but very different pathways. Furthermore, at the time, this is the only described MS-based method to combine the detection of $\mathrm{O}^{6}-\mathrm{CMG}$, $\mathrm{O}^{6}-\mathrm{MeG}, \mathrm{M} 1 \mathrm{G}$ and CroG.

DNA adductomics is an up-and-coming approach to investigate DNA adduct formation and its possible link to chronic disease. To elucidate supplementary meat or colon cancer related DNA adduct types and pathways, the use of this UHPLC-HRMS method was investigated in vitro and in vivo ensuring valid future clinical application in DNA adductomic studies. To the best of our knowledge, this paper describes 1 of only 3 high resolution MS-based DNA adductome mapping methods, whereas it is the first to explore and illustrate the use of Quadrupole-Orbitrap technology for DNA adduct profiling purposes.

Supplementary, an in-house database that lists known DNA adducts, was constructed and implemented to facilitate the complex process of omics data interpretation.

\subsection{DNA adduct analysis with UHPLC-HRMS/MS}

All relevant performance characteristics of the MS method were in accordance with internationally accepted analytical criteria. However, because of the method's intended use in a clinical setting, most attention was paid to the achievable LODs and LOQs for the 4 targeted DNA adducts prior to in vitro and/or in vivo SIM-HRMS/MS method application.

The in vivo relevance of the detection of $\mathrm{O}^{6}-\mathrm{CMG}$ has been demonstrated by its qualitative detection in several biological samples like blood, colon biopsies and exfoliated colonocytes [26-28]. To the best of our knowledge, average endogenous levels of $\mathrm{O}^{6}$-CMG in human tissue have not yet been reported. Therefore, at present, a scientifically based statement on the applicability of this method for the quantification of possibly low endogenous $\mathrm{O}^{6}$ CMG DNA adduct levels is not possible. Cupid et al. described the measurement of 35-80 $0^{6}$-CMG DNA adducts per $10^{8}$ nucleotides in blood of volunteers consuming a high meat diet [28]. These DNA adducts levels were detected by means of Immunoslot Blot with an LOD of 15 adducts per $10^{8}$ nucleotides, which confirms the competitiveness of our targeted SIM-HRMS/MS method.

A correct quantification of endogenous levels of the $0^{6}-\mathrm{MeG}$ DNA adduct with an LOQ of 2.82 adducts per $10^{8}$ nucleotides with MS/MS acquisition will depend on tissue or cell type. For example, Kang et al. reported the detection of less than one $0^{6}$-MeG DNA adduct in $10^{8}$ nucleotides in leucocyte DNA, whereas more than two $\mathrm{O}^{6}-\mathrm{MeG}$ adducts per $10^{8}$ nucleotides appeared to be present in hepatic DNA $[3,29]$. This suggests that our current method allows detection and quantification of $\mathrm{O}^{6}-\mathrm{MeG}$ in a clinically relevant range. If required, the current LOQ and LOD could be improved by tweaking the MS and HESI settings. However, at the time, all settings were optimized for the simultaneous detection of all 4 targeted DNA adducts and for $\mathrm{O}^{6}-\mathrm{CMG}$, demonstrating the highest LOQ and LOD, in particular.

The retrieved LOQs for M1G and CroG ( $<5$ adducts per $10^{8} \mathrm{nu}-$ cleotides) appeared to be more than sufficient. Indeed, according to previously reported endogenous levels of M1G and CroG in several tissue types, all reported DNA adduct levels exceeded 6 adducts per $10^{8}$ nucleotides [3], which implies that our LOQs are below endogenously present M1G and CroG DNA adduct levels.

\subsection{In vitro application}

The presence of both targeted and untargeted DNA adducts were investigated in blank CT-DNA or CT-DNA treated with genotoxic chemicals to demonstrate the in vitro application of the described method and the in-house constructed database.

In blank CT-DNA samples, different DNA adducts could be retrieved. M1G appeared to be the sole detected targeted DNA adduct endogenously present in CT-DNA and similar amounts of M1G in CT-DNA have been reported before [30]. Using the untargeted HRMS approach, the accurate masses of 20 additional compounds (Fig. 3) could be linked to DNA adducts included in the selfconstructed diet-related DNA adduct database (see Supporting information Table 1 ), resulting in a putative identification. The endogenous or exogenous occurrence of several of these DNA adducts in DNA and their link to for example oxidative stress [31,32], exposure to environmental carcinogens [33,34] and carcinogenesis $[32,35]$, have been reported previously, acknowledging their 
relevance.

Treatment of CT-DNA with KDA, MDA and CRO resulted in the formation and detection of the 4 targeted DNA adducts. The demonstrated dose response effect was to be expected for KDA, a well-known NOC, and both $\mathrm{O}^{6}-\mathrm{MeG}$ and $\mathrm{O}^{6}-\mathrm{CMG}$, since the methylation and carboxymethylation of deoxyguanosine at the $\mathrm{O}^{6}$ position by nitrosated glycine derivatives have been documented in the past $[19,36]$. The demonstrated formation of M1G in CT-DNA by attack of the $G$ base by the highly reactive lipid peroxidation product MDA has also been reported extensively in literature, with M1G being the predominantly formed DNA adduct [37]. The same applies for CRO and CroG formation [9]. The more 'efficient' and pronounced interaction of MDA and CRO with DNA to form M1G and CroG, compared to the interaction of KDA with DNA to form $\mathrm{O}^{6}$ CMG and $\mathrm{O}^{6}-\mathrm{MeG}$ (lower added MDA and CRO concentrations (compared to KDA) yield similar DNA adduct concentrations; Fig. 4) can be explained by the highly reactive nature of lipid peroxidation products [31].

Next to the detection of targeted DNA adducts, application of the HRMS method also enabled the detection of untargeted DNA adducts. Rapid putative identification of these compounds in retrospect was empowered by the use of the self-constructed database. In total, 25 untargeted DNA adducts were detected in blank CT-DNA and/or upon treatment with KDA, MDA and/or CRO. Several of these putatively identified DNA adducts have been detected previously and described in literature (Supporting information Table 1), acknowledging their relevance. Retrieval of these DNA adducts clearly emphasizes the potential of the HRMS method for DNA adductome mapping and the ease of putative DNA adduct identification by means of our DNA adduct database.

Some of the untargeted DNA adducts reported above have not been investigated extensively. However, considering the possible detection of all these compounds after incubation of DNA with KDA, MDA or CRO, further investigation is warranted. After all, extensive research on the possible link between toxic endo- and exogenous NOCs, lipid peroxidation products and disease has been conducted in the past and has revealed a connection to an increased cancer risk $[38,39]$.

\subsection{In vivo application}

The described DNA adductomics method has been constructed for future use in clinical studies. Therefore, preliminary tests for in vivo application were conducted on 10 colon tumor tissue samples. This resulted in the detection of the $0^{6}-\mathrm{CMG}$ DNA adduct in a vast majority of colon tumor biopsies. The other 3 targeted compounds could not be retrieved in any of the biopsy samples, although they have been detected in different human tissue types before [3], thus suggesting that M1G, CroG and $\mathrm{O}^{6}-\mathrm{MeG}$ were either absent or no longer present above the LOD in the analyzed biopsies. ToxID ${ }^{\mathrm{TM}}$ screening revealed the likely presence of 17 untargeted DNA adducts of which some could be retrieved in all 10 biopsies, whilst others could only be detected in some or just one sample(s).

Current knowledge on the stability of different DNA adducts is still limited. In consequence, future DNA adduct profiling should be carried out as soon as possible following tissue sampling to avoid DNA adduct instability issues. As these possible stability issues were not sufficiently taken into account during colon biopsy sample handling and storage, any conclusions obtained from the earlier reported results on the possible connection between observed DNA adduct levels and diet or disease status would be too precarious at the time being. A more extensive discussion on DNA adduct profiling and its link to diet and disease status will therefore be conducted during extensive follow-up studies. Nonetheless, the described DNA adductomics method already proved its usefulness as both targeted and putative untargeted diet-related DNA adducts were detected easily in vivo. Moreover, this is the first paper to report DNA adductome mapping of (tumoral) colonic tissue.

To provide an overview, the envisioned workflow for DNA adduct profiling in future DNA adductomic studies by means of the reported UHPLC-HRMS(/MS) method is documented in the graphical abstract.

\subsection{The merit of a DNA adduct database}

The human diet is quite complex and person-dependent, which implies that the impact of the individual human diet is very hard to pin to down. As diet and lifestyle significantly contribute to colon cancer risk, further investigation is warranted to link dietary toxicity to chronic disease through DNA adduct formation. Different diet-related DNA adduct types can be formed by alkylation and oxidation or mycotoxin, heterocyclic amine (HCA), PAH, acrylamide exposure, etc. In this context, a total of 123 different DNA adducts have currently been listed in an in-house (diet-related) DNA adduct database. Most of the listed DNA adducts have already been investigated in vitro or in vivo in relation to one or multiple 'suspicious' foodstuffs (see Supporting information Table 1 for detailed references). As the currently available information concerning the genotoxic effects of different food constituents is quite elaborate, yet still inconclusive, and since the use of DNA adductomics is still under development, it may be stated that although our DNA adduct list (Supporting information Table 1) is already quite extensive, it is not definite. Nevertheless, due to the complex nature of the human diet and the long list of related research questions, the database can definitely help expose relevant DNA adduct types that require a more extensive investigation.

As different heterocyclic amine, polycyclic aromatic hydrocarbon, mycotoxin and acrylamide generated DNA adducts were also included in the database, the untargeted UHPLC-HRMS approach and database can also be put to use in related research that focuses on other diet-related chronic diseases. Furthermore, the selfconstructed database includes a multitude of DNA adducts generated by unspecific alkylation and oxidation reactions which could be caused by several types of genotoxic, mutagenic and carcinogenic chemicals.

\section{Conclusion}

Hybrid HRMS systems yield highly specific information on compound mass, elemental composition and identity, whilst also enabling study of fragmentation patterns offering several advantages regarding structural elucidation. In consequence, such MS systems are particularly well suited for DNA adductomics $[12,40]$. As it is our goal to implement the described method in the search for DNA adduct formation through dietary exposure to genotoxic chemicals in the meat-cancer relationship, the newly developed and successfully validated UHPLC-HRMS(/MS) method currently combines the simultaneous detection of 4 structurally and chemically different DNA adducts. The obtained LODs and LOQs for the targeted detection of $\mathrm{O}^{6}-\mathrm{CMG}, \mathrm{O}^{6}-\mathrm{MeG}, \mathrm{M} 1 \mathrm{G}$ and CroG with SIMMS/MS allow in vitro and in vivo application. The state-of-the-art hybrid MS method also showed great promise for untargeted DNA adduct detection in future DNA adductomic studies as the Orbitrap revealed the presence of several putatively identified DNA adducts in different DNA samples. This confirms the applicability of the untargeted full scan HRMS approach and its envisioned use for DNA adductome mapping. The use of the in-house DNA-adduct database in research focusing on diet and lifestyle related chronic diseases could expedite exposure of relevant biomarkers and provide new insights in disease etiology and prevention. The described 
adductomics methodology and database will thus serve as a basis for the analysis of both endogenous and exogenous DNA adducts in DNA from several tissue types and for a wide variety of research topics.

\section{Acknowledgments}

The authors would like to thank D. Stockx and Dr. J. Van Hende for their practical assistance and expertise. Special thanks go to J.A.L. Kiebooms.

\section{Appendix A. Supplementary data}

Supplementary data related to this article can be found at http:// dx.doi.org/10.1016/j.aca.2015.08.019.

\section{References}

[1] M.C. Poirier, Chemical-induced DNA damage and human cancer risk, Nat. Rev Cancer 4 (2004) 630-637.

[2] P.B. Farmer, DNA and protein adducts as markers of genotoxicity, Toxicol. Lett. 149 (2004) 3-9.

[3] R. De Bont, N. van Larebeke, Endogenous DNA damage in humans: a review of quantitative data, Mutagenesis 19 (2004) 169-185.

[4] G.S. Qian, R.K. Ross, M.C. Yu, J.M. Yuan, Y.T. Gao, B.E. Henderson, G.N. Wogan, J.D. Groopman, A follow-up study of urinary markers of aflatoxin exposure and liver cancer risk in Shanghai, People's Republic of China, Cancer Epidemiol. Biomark. Prev. 3 (1994) 3-10. A publication of the American Association for Cancer Research, cosponsored by the American Society of Preventive Oncology.

[5] J. Angerer, U. Ewers, M. Wilhelm, Human biomonitoring: state of the art, Int. J. Hyg. Environ. Health 210 (2007) 201-228.

[6] G.G. Kuhnle, S.A. Bingham, Dietary meat, endogenous nitrosation and colorectal cancer, Biochem. Soc. Trans. 35 (2007) 1355-1357.

[7] V.L. Go, D.A. Wong, R. Butrum, Diet, nutrition and cancer prevention: where are we going from here? J. Nutr. 131 (2001) 3121s-3126s.

[8] D.E. Corpet, Red meat and colon cancer: should we become vegetarians, or can we make meat safer? Meat Sci. 89 (2011) 310-316.

[9] L.J. Marnett, Oxy radicals, lipid peroxidation and DNA damage, Toxicology 181-182 (2002) 219-222.

[10] M.W. Himmelstein, P.J. Boogaard, J. Cadet, P.B. Farmer, J.H. Kim, E. A. Martin, R. Persaud, D.E. Shuker, Creating context for the use of DNA adduct data in cancer risk assessment: II. Overview of methods of identification and quantitation of DNA damage, Crit. Rev. Toxicol. 39 (2009) 679-694.

[11] P.B. Farmer, R. Singh, Use of DNA adducts to identify human health risk from exposure to hazardous environmental pollutants: the increasing role of mass spectrometry in assessing biologically effective doses of genotoxic carcinogens, Mutat. Res. Rev. Mutat. Res. 659 (2008) 68-76.

[12] S. Balbo, R.J. Turesky, P.W. Villalta, DNA adductomics, Chem. Res. Toxicol. 27 (2014) 356-366.

[13] H. Koc, J.A. Swenberg, Applications of mass spectrometry for quantitation of DNA adducts, J. Chromatogr. B 778 (2002) 323-343.

[14] S. Balbo, S.S. Hecht, P. Upadhyaya, P.W. Villalta, Application of a highresolution mass-spectrometry-based DNA adductomics approach for identification of DNA adducts in complex mixtures, Anal. Chem. 86 (2014) $1744-1752$.

[15] J.M. Gavina, C. Yao, Y.L. Feng, Recent developments in DNA adduct analysis by mass spectrometry: a tool for exposure biomonitoring and identification of hazard for environmental pollutants, Talanta 130 (2014) 475-494.

[16] B.H. Monien, Mass spectrometric DNA adduct quantification by multiple reaction monitoring and its future use for the molecular epidemiology of cancer, Adv. Exp. Med. Biol. 806 (2014) 383-397.

[17] X. Liu, Z. Ser, J.W. Locasale, Development and quantitative evaluation of a high-resolution metabolomics technology, Anal. Chem. 86 (2014) 2175-2184.

[18] C. Xie, D. Zhong, K. Yu, X. Chen, Recent advances in metabolite identification and quantitative bioanalysis by LC-Q-TOF MS, Bioanalysis 4 (2012) 937-959.

[19] J. Vanden Bussche, S.A. Moore, F. Pasmans, G.G. Kuhnle, L. Vanhaecke, An approach based on ultra-high pressure liquid chromatography-tandem mass spectrometry to quantify 06-methyl and 06-carboxymethylguanine DNA adducts in intestinal cell lines, J. Chromatogr. A 1257 (2012) 25-33.

[20] E. Gottschalg, G.B. Scott, P.A. Burns, D.E. Shuker, Potassium diazoacetateinduced p53 mutations in vitro in relation to formation of O6carboxymethyl- and 06-methyl-2'-deoxyguanosine DNA adducts: relevance for gastrointestinal cancer, Carcinogenesis 28 (2007) 356-362.

[21] US Food and Drug Administration, Draft Guidance for Industry for Bioanalytical Method Validation, 2013. www.fda.gov.

[22] US Food and Drug Administration, Guidelines for the Validation of Chemical Methods for the FDA Foods Program, 2012. www.fda.gov.

[23] The Commision of the European Communities, Commission Decision of 12 August 2002 Implementing Council Directive 96/23/EC Concerning the Performance of Analytical Methods and the Interpretation of Results, 2002. eurlex.europa.eu.

[24] A. Pfohl-Leszkowicz, Y. Grosse, V. Carriere, P.H. Cugnenc, A. Berger, F. Carnot, P. Beaune, I. de Waziers, High levels of DNA adducts in human colon are associated with colorectal cancer, Cancer Res. 55 (1995) 5611-5616.

[25] J. Vanden Bussche, L.Y. Hemeryck, T. Van Hecke, G.G.C. Kuhnle, F. Pasmans, S.A. Moore, T. Van de Wiele, S. De Smet, L. Vanhaecke, O6carboxymethylguanine DNA adduct formation and lipid peroxidation upon in vitro gastrointestinal digestion of haem-rich meat, Mol. Nutr. Food Res. 58 (9) (2014) 1883-1896.

[26] M.H. Lewin, N. Bailey, T. Bandaletova, R. Bowman, A.J. Cross, J. Pollock, D.E.G. Shuker, S.A. Bingham, Red meat enhances the colonic formation of the DNA adduct 06-carboxymethyl guanine: implications for colorectal cancer risk, Cancer Res. 66 (2006) 1859-1865.

[27] C.N. Hall, A.F. Badawi, P.J. O'Connor, R. Saffhill, The detection of alkylation damage in the DNA of human gastrointestinal tissues, Br. J. Cancer 64 (1991) 59-63.

[28] B.C. Cupid, Z. Zeng, R. Singh, D.E. Shuker, Detection of O6-carboxymethyl-2' deoxyguanosine in DNA following reaction of nitric oxide with glycine and in human blood DNA using a quantitative immunoslot blot assay, Chem. Res. Toxicol. 17 (2004) 294-300.

[29] H.-I. Kang, C. Konishi, T. Kuroki, N.-h. Huh, Detection of O6-methylguanine, 04-methylthymine and 04-ethylthymine in human liver and peripheral blood leukocyte DNA, Carcinogenesis 16 (1995) 1277-1280.

[30] Y.C. Jeong, J.A. Swenberg, Formation of M1G-dR from endogenous and exogenous ROS-inducing chemicals, Free Radic. Biol. Med. 39 (2005) 1021-1029.

[31] L.J. Marnett, Oxyradicals and DNA damage, Carcinogenesis 21 (2000) $361-370$.

[32] A. Valavanidis, T. Vlachogianni, C. Fiotakis, 8-hydroxy-2' -deoxyguanosine (8$\mathrm{OHdG}$ ): a critical biomarker of oxidative stress and carcinogenesis, J. Environ. Sci. Health Part C Environ. Carcinog. Ecotoxicol. Rev. 27 (2009) 120-139.

[33] G. Cheng, M. Wang, P.W. Villalta, S.S. Hecht, Detection of 7-(2'-Carboxyethyl) guanine but not 7-carboxymethylguanine in human liver DNA, Chem. Res, Toxicol. 23 (2010) 1089-1096.

[34] S.S. Mirvish, A.E. Ross, B. Gold, N. Drake, In vitro and in vivo formation of 7-(2'carboxyethyl)guanine from the liver carcinogen 1-nitroso-5,6-dihydrouracil and its reactions with water and methanol, J. Natl. Cancer Inst. 74 (1985) 1105-1110.

[35] B. Tudek, E. Speina, Oxidatively damaged DNA and its repair in colon carcinogenesis, Mutat. Res. 736 (2012) 82-92.

[36] D.E.G. Shuker, G.P. Margison, Nitrosated glycine derivatives as a potential source of O6-methylguanine in DNA, Cancer Res. 57 (1997) 366-369.

[37] L.J. Marnett, Lipid peroxidation-DNA damage by malondialdehyde, Mutat. Res. 424 (1999) 83-95.

[38] W. Lijinsky, N-Nitroso compounds in the diet, Mutat. Res. Genet. Toxicol. Environ. Mutagen. 443 (1999) 129-138.

[39] H. Bartsch, J. Nair, Chronic inflammation and oxidative stress in the genesis and perpetuation of cancer: role of lipid peroxidation. DNA damage, and repair, Langenbeck's Arch. Surg. dtsch. Ges. fur Chir. 391 (2006) 499-510.

[40] N. Tretyakova, P.W. Villalta, S. Kotapati, Mass spectrometry of structurally modified DNA, Chem. Rev. 113 (2013) 2395-2436. 